\title{
Aquaporin-2 water channel mutations and nephrogenic diabetes insipidus: new variations on a theme
}

\author{
Jonas Rutishauser and Peter Kopp \\ Division of Endocrinology, Metabolism and Molecular Medicine, Northwestern University, Chicago, Illinois 60611, USA \\ (Correspondence should be addressed to J Rutishauser, Department of Internal Medicine, Kantonsspital, 6000 Lucerne, Switzerland)
}

The aquaporins are a family of water channels with currently nine cloned mammalian members (1). At least four of these proteins are involved in renal water transport, and three aquaporins are expressed in the principal cells of the collecting duct. Aquaporin-2 (AQP2) appears to be essential in the vasopressindependent reabsorption of water and in concentration of urine (Fig. 1). In the absence of vasopressin (AVP), AQP2 is located as a homotetrameric molecule in subapical storage vesicles. Vasopressin, secreted from the posterior pituitary in response to hypovolemia and/ or hypernatremia, binds to the vasopressin type 2 receptor (AVPR2), a G protein-coupled seven transmembrane domain receptor that is expressed in the basolateral membrane of collecting duct cells. Activation of the AVPR2 by its ligand stimulates cAMP formation and phosphorylation of protein kinase A which subsequently elicits further cytosolic and nuclear events. Phosphorylation of AQP2 leads to fusion of the storage vesicles with the luminal cell membrane and results in reabsorption of water from the tubular lumen along the osmotic gradient. At the basolateral membrane, aquaporin-3 and -4 facilitate water transport into the bloodstream. After dissociation of AVP from its receptor, AQP2 is removed from the luminal membrane by endocytosis, thus decreasing the water permeability of the renal collecting duct epithelium (Fig. 1) (1). Besides this rapid effect of AVP, activation of the cAMP cascade also results in increased transcription of the AQP2 gene which contains a cAMP responsive element in its promoter (2).

Nephrogenic diabetes insipidus (NDI) is most commonly acquired, but the inability of the kidney to concentrate urine in response to AVP may also occur as an inherited disorder. The more prevalent X-linked form is caused by loss-of-function mutations in the AVPR2 (3). Since the AVPR2 also mediates the AVPinduced increase in the von Willebrand factor and Factor VIII, this response is absent or blunted in patients with X-linked NDI. A subset of patients with inherited NDI has, however, a normal increase in these coagulation factors in response to DDAVP, and the mode of transmission is not X-linked. In 1994, Deen et al. demonstrated compound heterozygosity for inactivating mutations in the AQP2 gene located on chromosome $12 \mathrm{q} 12-13$ in a patient with autosomal recessive NDI (4). During the last four years, further families with autosomal recessive NDI and AQP2 mutations have been characterized, and functional studies have provided evidence that the detected mutations inactivate the protein (5-9).

A novel variant of familial NDI has now been characterized by Mulders et al. who reported a family with NDI caused by a dominant mutation in the AQP2 gene (10). They studied a female patient with congenital NDI and her daughter who presented with progressive polyuria and polydipsia during childhood. Sequence analysis revealed a normal AVPR2 gene, but the presence of a heterozygous de novo transition of G866 to A in the coding sequence of the AQP2 gene in the mother. This point mutation results in the substitution of glutamic acid 258 by lysine (Glu258Lys) in the intracellular carboxyterminus of the protein. The mutation was also found in the affected daughter, suggesting an autosomal dominant mode of transmission of the NDI phenotype. Since only one allele was affected, the question arose as to how the mutant allele exerts a dominant negative effect over the wild type. The Glu258Lys mutation is in close vicinity to serine 256 which is phosphorylated by protein kinase A, a step that appears to be critical for fusion of AQP2-containing vesicles with the apical plasma membrane. Phosphorylation of serine 256 was, however, not affected by the Glu258Lys mutation. Concomitant expression of AQP2 wild type and the Glu258Lys mutant in Xenopus oocytes then confirmed a dominant negative effect of the mutant over the wild type AQP2; oocytes expressing both the wild type and the mutant protein showed decreased water permeability in comparison to oocytes expressing wild type AQP2 alone or an AQP2 mutant (Arg187Cys) associated with autosomal recessive NDI. Immunoblot experiments demonstrated that the Glu258Lys mutant was only weakly expressed in the plasma membrane in comparison to wild type AQP2. Immunofluorescence and immunogold labeling revealed that the mutant protein was retained in the Golgi apparatus. This was also the case in oocytes expressing both the mutant and the wild type, suggesting that the dominant negative effect of the Glu258Lys mutant occurs through Golgi retention of AQP-2 tetramers composed of mutant and wild type molecules.

The findings obtained with this dominant AQP2 mutant contrast with the functional consequences of AQP2 mutations causing autosomal recessive NDI. The 


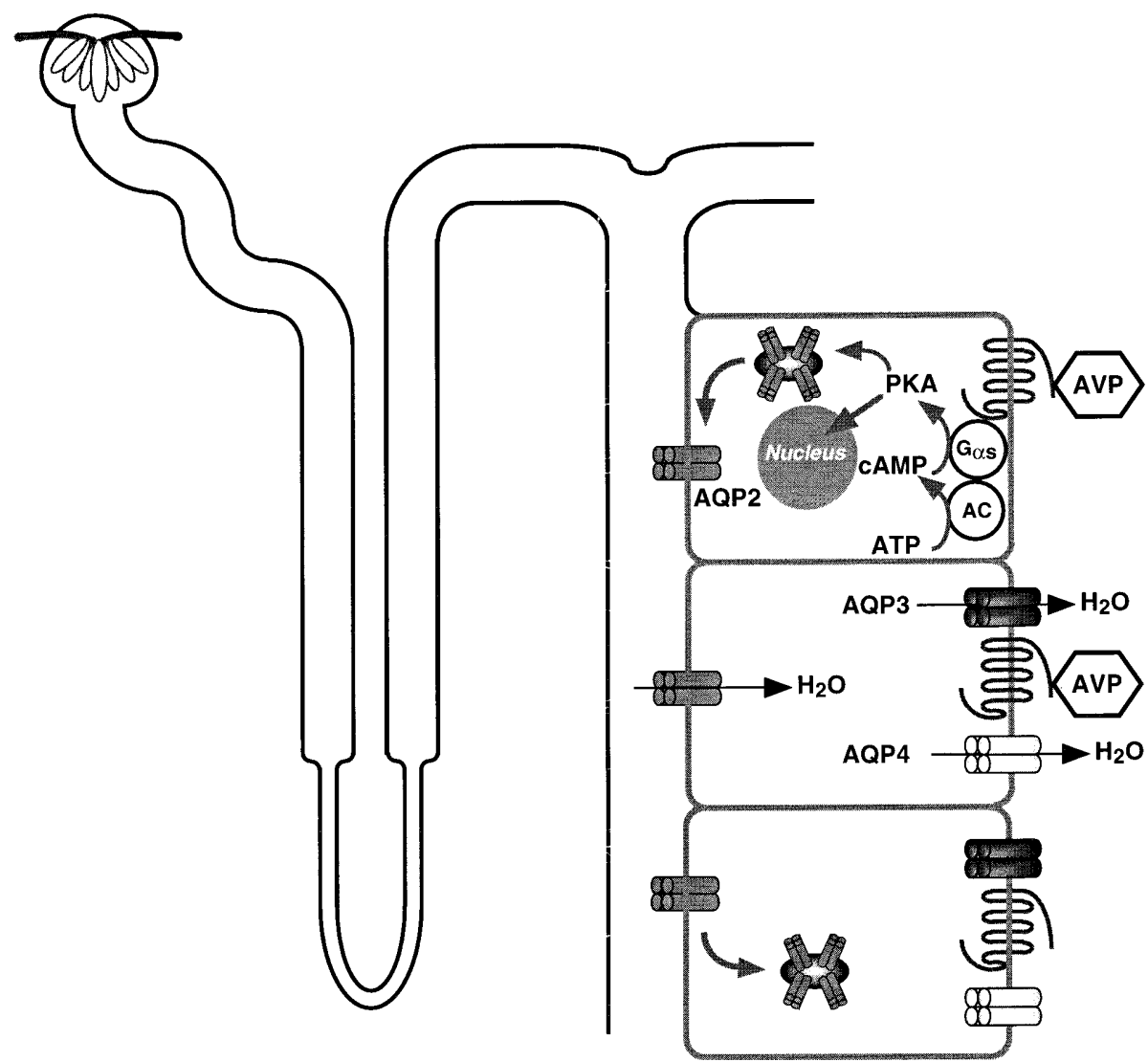

Figure 1 Regulation of aquaporin-2 (AQP2) in principal cells of the renal collecting duct. After binding of AVP to the AVPR2, the activation of Gs $\alpha$ and adenylyl cyclase (AC) results in the formation of intracellular cAMP and phosphorylation of protein kinase A (PKA). AQP2 is stored as a homotetramer in subapical vesicles. Phosphorylation of AQP2 by PKA leads to fusion of the vesicles with the luminal cell membrane, resulting in reabsorption of water from the tubular lumen. PKA also activates nuclear factors which increase AQP2 transcription. At the basolateral membrane, aquaporin-3 and -4 facilitate water transport into the bloodstream. After dissociation of AVP from its receptor, AQP2 is removed from the luminal membrane by endocytosis.

cellular mechanisms resulting in loss of function of five recessive AQP2 mutations were recently further characterized in a remarkable study by Tamarappoo and Verkman (11). These authors assessed water permeability in Xenopus oocytes, as well as protein processing and targeting in Chinese hamster ovary $(\mathrm{CHO})$ and Madin-Darby canine kidney (MDCK) cells. After correction for membrane expression levels, the oocyte water permeability was significantly decreased in one mutant (Arg187Cys), but probably not significantly impaired in the three other mutants (Leu22Val, Thr126Met, Ala147Thr). In a further mutant, Cys181Trp, water permeability could not be assessed because it was not expressed in the plasma membrane. Metabolic labeling studies in transiently transfected $\mathrm{CHO}$ cells showed a significantly decreased stability of Ala147Thr, and only slightly reduced stability for Arg187Cys and Thr126Met. In immunofluorescence and immunoblot experiments wild type AQP2 was detectable in the cell membrane and endosomal compartments. In contrast, all mutant proteins were retained in the endoplasmic reticulum
(ER), albeit to various degrees. Remarkably, the addition of chemical chaperones (glycerol, dimethylsulfoxide, trimethylamine oxide), substances that promote proper folding of proteins in the ER, led to a redistribution of most mutants to the membrane and endosomes. Water permeability measurements also demonstrated functional rescue of one mutant (Thr126Met) in transiently transfected glycerol-treated CHO cells (11).

What is the significance of these studies? The report by Mulders et al. (10) demonstrates that NDI can also be inherited in an autosomal dominant fashion, an observation which is of direct clinical relevance. Moreover, this study suggests that the dominant mutant is associated with retention of AQP2 tetramers in the Golgi apparatus, a finding which contrasts with the retention of the recessive mutants in the ER. NDI caused by AQP2 mutations thus belongs to the large group of protein folding diseases characterized by altered trafficking of mutant proteins. This group includes, among many others, disorders as diverse as Alzheimer's disease, $\alpha_{1}$ proteinase inhibitor deficiency, some forms of retinitis 
pigmentosa, and cystic fibrosis (12). In particular, the study by Tamarappoo and Verkman (11) suggests that at least a subset of mutant AQP2 water channels may fail to interact properly with molecular chaperones, proteins that assist the molecule in attaining its tertiary structure that is required for correct targeting. The in vitro rescue with chemical chaperones demonstrated for recessive AQP2 mutations has also been achieved with mutant cystic fibrosis transmembrane conductance regulator (CFTR) proteins associated with cystic fibrosis (13). These observations may thus be of relevance for the development of novel forms of treatment of such disorders.

Lastly, it is also of interest to compare the molecular mechanism underlying NDI caused by AQP 2 mutations to the autosomal dominant neurohypophyseal form of diabetes insipidus which is caused by mutations in the vasopressin-neurophysin II (AVP-NPII) gene. While mutant AVP-NPII proteins are also retained in the ER $(14,15)$, the dominant phenotype of this disease appears to result from a distinct mechanism. It is thought to be the consequence of a progressive degeneration of AVP-producing hypothalamic neurons caused by a neurotoxic effect of the mutant protein (15, 16). This mechanism provides an explanation for the gradual development of polyuria and polydipsia during childhood, which contrasts with the typically severe, congenital onset of hereditary NDI. So far, autosomal recessive neurohypophyseal diabetes insipidus has not been reported in humans. In the Brattleboro rat, a recessive mutation in the AVP-NPII precursor leads to a frameshift with an extended carboxyterminus which impedes processing and secretion of the protein $(17,18)$.

Further genetic heterogeneity, i.e. defects in still other genes, may well be discovered in familial neurohypophyseal and nephrogenic diabetes insipidus in the future. In addition, the pending characterization of the various forms of hereditary diabetes insipidus in transgenic animals will undoubtedly provide more detailed insights into the pathogenesis of these disorders, and they will allow testing of novel therapeutic strategies in vivo.

\section{Acknowledgements}

We are grateful to Dr Gary L Robertson, Northwestern University, Chicago for stimulating discussions on all aspects of diabetes insipidus. JR is supported by a fellowship from the Kantonale Nachwuchskommission des Kantons Zürich and the Schweizerische Stiftung für Medizinisch-Biologische Stipendieu, PK by a Northwestern University New Investigator Award from the Howard Hughes Medical Institute.

\section{References}

1 King LS \& Agre P. Pathophysiology of the aquaporin water channels. Annual Review of Physiology 199658 619-648.
2 Hozawa S, Holtzman EJ \& Ausiello DA. cAMP motifs regulating transcription in the aquaporin-2 gene. American Journal of Physiology 199639 C1695-C1702.

3 Bichet D. Nephrogenic diabetes insipidus and vasopressin receptor mutations. In G Proteins, Receptors, and Disease, pp 167-179. Ed AM Spiegel. Totowa: Humana Press, 1998.

4 Deen PM, Verdijk MA, Knoers NVAM, Wieringa B, Monnens LA, van $\mathrm{Os} \mathrm{CH}$ et al. Requirement of human renal water channel aquaporin-2 for vasopressin-dependent concentration of urine. Science $199426492-95$.

5 van Lieburg AF, Verdijk MA, Knoers VV, van Essen AJ, Proesmans W, Mallmann R et al. Patients with autosomal nephrogenic diabetes insipidus homozygous for mutations in the aquaporin-2 water-channel gene. American Journal of Human Genetics 199455 648-652.

6 Deen PM, Croes H, van Aubel RA, Ginsel LA \& van Os CH. Water channels encoded by mutant aquaporin-2 genes in nephrogenic diabetes insipidus are impaired in their cellular routing. Journal of Clinical Investigation 199595 2291-2296.

7 Oksche A, Moller A, Dickson J, Rosendahl W, Rascher W, Bichet DG et al. Two novel mutations in the aquaporin-2 and the vasopressin V2 receptor genes in patients with congenital nephrogenic diabetes insipidus. Human Genetics $199698587-$ 589.

8 Hochberg Z, Van Lieburg A, Even L, Brenner B, Lanir N, Van Oost BA et al. Autosomal recessive nephrogenic diabetes insipidus caused by an aquaporin-2 mutation. Journal of Clinical Endocrinology and Metabolism 199782 686-689.

9 Mulders SM, Knoers NV, Van Lieburg AF, Monnens LA, Leumann $\mathrm{E}$, Wuhl E et al. New mutations in the AQP2 gene in nephrogenic diabetes insipidus resulting in functional but misrouted water channels. Journal of the American Society of Nephrology 19978 242-248.

10 Mulders SM, Bichet DG, Rijss JPL, Kamsteeg E-J, Arthus M-F, Lonergan $\mathrm{M}$ et al. An aquaporin-2 water channel mutant which causes autosomal dominant nephrogenic diabetes insipidus is retained in the Golgi complex. Journal of Clinical Investigation 1998 $10257-66$.

11 Tamarappoo BK \& Verkman AS. Defective aquaporin-2 trafficking in nephrogenic diabetes insipidus and correction by chemical chaperones. Journal of Clinical Investigation $19981012257-$ 2267.

12 Thomas PJ, Qu BH \& Pedersen PL. Defective protein folding as a basis of human disease. Trends in Biochemical Science 199520 456-459.

13 Brown CR, Hong-Brown LO, Biwersi J, Verkman AS \& Welch WJ. Chemical chaperones correct the mutant phenotype of the delta F508 cystic fibrosis transmembrane conductance regulator protein. Cell Stress Chaperones 19961 117-125.

14 Olias G, Richter D \& Schmale H. Heterologous expression of human vasopressin-neurophysin precursors in a pituitary cell line: defective transport of a mutant protein from patients with familial diabetes insipidus. DNA and Cell Biology 199615 929-935.

15 Ito M, Jameson JL \& Ito M. Molecular basis of autosomal dominant neurohypophyseal diabetes insipidus. Cellular toxicity caused by the accumulation of mutant vasopressin precursors within the endoplasmic reticulum. Journal of Clinical Investigation 199799 1897-1905.

16 Rittig S, Robertson GL, Siggaard C, Kovacs L, Gregersen N, Nyborg $\mathrm{J}$ et al. Identification of 13 new mutations in the vasopressinneurophysin II gene in 17 kindreds with familial autosomal dominant neurohypophyseal diabetes insipidus. American Journal of Human Genetics 199658 107-117.

17 Schmale H \& Richter D. Single base deletion in the vasopressin gene is the cause of diabetes insipidus in Brattleboro rats. Nature 1984308 705-709.

18 Kim JK, Summer SN, Wood WM, Brown JL \& Schrier RW. Arginine vasopressin secretion with mutants of wild-type and Brattleboro rats AVP gene. Journal of the American Society of Nephrology 19978 1863-1869. 